\title{
Oncogenes, reductionism and all that
}

\section{Much of the excitement generated of late by studies of human oncogenes stems from the belief that the causation of cancer will be better understood. But is that not naive reductionism? Simply: NO}

THE excitement in the past few months about the oncogenes, especially that generated by the discovery (see Nature 300, 143 and $149 ; 1982$ ) that a single point mutation in a gene coding for a protein called p21 appears to characterize some human carcinomas, has now predictably begun to stimulate scepticism. Naturally, in such a novel field of enquiry, there is a host of technical questions to be asked. Is the occurrence of the oncogenes now thought to be associated with malignancy confined to overtly malignant cells? And to what extent do the special properties of the NIH 3T3 cells so far used as an assay of malignancy predetermine some of the results so far obtained? Such questions are plainly important, but the answers in due course provided for them are unlikely to diminish the central importance of the recognition that there is, at last, an identifiable material, the protein p21, whose structure appears to be intimately involved in the difference between normalcy and malignancy. But can a biological phenomenon as complicated as cancer be so simply explained? Is it not outrageously reductionist to suppose that a molecular account can be given of a biological aberration known to involve many kinds of cellular interactions as well as environmental and even psychological influences?

\section{Reductionism}

That question has not yet been fully debated in Nature. No doubt, in due course, it will be. The question was, however, raised the other day by a correspondent who had also written similarly to another journal. (Ironically, it is understood that the letter is to be published in Science in spite of that journal's recent somewhat pompous strictures against authors seeking several outputs for a single manuscript.) What follows should therefore either be read as an anticipatory response to the argument that reductionists have no business bothering their heads with complicated questions such as the causation of cancer or should be regarded (in the British idiom) as an Aunt Sally, a gratuitous target of opportunity (in another idiom). The issue is nevertheless important, not merely because the problem of cancer is socially important but also because it bears directly on the design of an agenda for scientific enquiries of all kinds.

The public and sometimes even the professional image of the reductionist is not flattering, a little like that of the outand-out behaviourist among psychologists. A reductionist is one whose objective is to account for even the most complicated phenomena in terms of elementary interactions between more or less elementary entities. Elsewhere, among cosmologists for example, they may be people who worry about the phase changes that may account for the transition from a world in which free quarks exist to one in which all quarks are bound together in nucleons or sometimes mesons. In biology, reductionists appear as molecular biologists.

Everywhere, reductionists are intellectually vulnerable in three particular respects. First, and almost trivially, many obvious problems may lie technically beyond the reach of the reductionists: these days, it is a simple matter to calculate the electronic energy levels of a hydrogen atom, but simple molecules such as anthraquinone remain inaccessible except to those willing to include enough arbitrary constants in their calculations. Second, and more important, the reductionist agenda is no substitute for discovery: even in well trodden fields of physics such as the theory of wave motion, for example, it was not until recently appreciated that wave propagation can accommodate the motion of the apparently integral and unchanging packets of disturbance now called solitons. Is it any wonder that reductionist molecular biologists failed to predict reverse transcriptase, or the occurrence of introns in mammalian genes? Sensible reductionists, of course, acknowledge that their goal is not to reduce the whole of science to the definition of a few interparticle interactions and a mass of computation; the value of this way of looking at the world is rather to help more accurately to define the constantly moving boundary between what may be described as 'known' and the rest. Finally, reductionists are constantly exposed to the more subtle complaint that there are some phenomena so intricate, subtle and beyond the range of current concepts, that attempts to account for them serve only to demonstrate the arrogance, even foolishness, of those who try. Quite apart from the now discredited vitalists, reputable biologists belong in this third camp of critics. "Give us", they might say, "an atomic theory of memory and we might change our minds,"
The trouble with these arguments is that they are largely semantic. The difficulties of calculating the energy levels of anthraquinone do not undermine belief in the quantum theory but provide frequently convenient gibes, especially for those eager to suggest that some conceptual questions in science are forever inaccessible to the reductionists. But the motives by which those suggestions are inspired are themselves suspect. In relation to cancer, for example, it is often (and rightly) said that the most obvious characteristic of a cancer cell is that its specific differentiation has been to some extent unwound, so that it becomes an anarchic component of the tissue to which it belongs and also capable of finding a lodging in some quite different tissue. But then, the argument continues, molecular biologists have nothing tangible to say about differentiation (which is broadly speaking true). So how can they pretend that the time will soon arrive when a molecular explanation of malignancy is possible? Since, so far as can be told from our correspondent, the gibe stems not from a devotion to vitalism nor in a belief in other literally supernatural phenomena, the answer can be only another gibe "Let's wait and see".

\section{Balance}

Meanwhile, it is important that the status of the reductionist agenda in relation to oncogenes should not be exaggerated. It would be folly to suggest that the time will ever come when the relationship between malignancy and molecular events is so well known and so predictable that oncologists will become people skilled only in the techniques of genetic manipulation. On the record of the past few centuries, but especially since Friedrich Wöhler found that ammonium cyanate will isomerize into urea, however, it seems risky to suppose that there are phenomena of any kind inaccessible to explanation in terms of simpler phenomena. And meanwhile, the practical issue between the reductionists and their critics is the all-important question of how the balance will be struck, in the understanding of cancer, especially as illuminated by what is being learned about oncogenes, between unexpected empirical discovery and the necessarily limited predictive power of the reductionists. The chances are that, as always, empirical discovery will make the running but that the causation of malignancy will be understood, possibly even dealt with. 